\title{
Pre-operative CT scan measurements for predicting complications in patients undergoing complex ventral hernia repair using the component separation technique
}

\author{
H. Winters ${ }^{1,2}$ (D) L. Knaapen ${ }^{2}$ - O. R. Buyne ${ }^{2} \cdot$ S. Hummelink ${ }^{1} \cdot$ D. J. O. Ulrich ${ }^{1} \cdot$ H. van Goor ${ }^{2} \cdot$ E. van Geffen ${ }^{3}$. \\ N. J. Slater ${ }^{1,3}$
}

Received: 31 May 2018 / Accepted: 22 January 2019 / Published online: 7 March 2019

(c) The Author(s) 2019

\begin{abstract}
Background The component separation technique (CST) is considered an excellent technique for complex ventral hernia repair. However, postoperative infectious complications and reherniation rates are significant. Risk factor analysis for postoperative complication and reherniation has focused mostly on patient history and co-morbidity and shows equivocal results. The use of abdominal morphometrics derived from CT scans to assist in risk assessment seems promising. The aim of this study is to determine the predictability of reherniation and surgical site infections (SSI) using pre-operative CT measurements. Methods Electronic patient records were searched for patients who underwent CST between 2000 and 2013 and had a preoperative CT scan available. Visceral fat volume (VFV), subcutaneous fat volume (SFV), loss of domain (LOD), rectus thickness and width (RT, RW), abdominal volume, hernia sac volume, total fat volume (TFV), sagittal distance (SD) and waist circumference (WC) were measured or calculated. Relevant variables were entered in multivariate regression analysis to determine their effect on reherniation and SSI as separate outcomes.

Results Sixty-five patients were included. VFV $(p=0.025, \mathrm{OR}=1.65)$ was a significant predictor regarding reherniation. Hernia sac volume $(p=0.020, \mathrm{OR}=2.10)$ and SFV per $1000 \mathrm{~cm}^{3}(p=0.034, \mathrm{OR}=0.26)$ were significant predictors of SSI. Conclusion Visceral fat volume, subcutaneous fat volume and hernia sac volume derived from CT scan measurements may be used to predict reherniation and SSI in patients undergoing complex ventral hernia repair using CST. These findings may aid in optimizing patient-tailored preoperative risk assessment.
\end{abstract}

Keywords Complex ventral hernia $\cdot$ Component separation technique $\cdot$ Ramirez $\cdot$ BMI $\cdot$ Fat volume $\cdot$ Bodymorphometrics $\cdot$ Reherniation

Presented at the International Hernia Congress of the American Hernia Society, in Miami, America, March 12 through 15, 2018.

H. Winters

harm.winters@radboudumc.nl

1 Department of Plastic and Reconstructive Surgery, Radboud University Medical Center, PO Box 9101, 6500 HB Nijmegen, The Netherlands

2 Department of Surgery, Radboud University Medical Center, PO Box 9101, 6500 HB Nijmegen, The Netherlands

3 Department of Surgery, Jeroen Bosch Hospital, PO Box 90153, 5200 ME 's Hertogenbosch, The Netherlands

\section{Introduction}

Complex ventral hernia repair remains a very challenging domain for general and reconstructive surgeons. Synthetic mesh bridging is used for small ventral hernias but coincides with a high reherniation rate and bulging of the abdomen when used for large and more complex defects and should be avoided in these cases [1-3]. The component separation technique (CST) is considered an excellent technique to close large complex defects as this procedure reestablishes the abdominal wall integrity. In this procedure, the external oblique muscles are released to facilitate the sliding of these myofascial flaps to allow re-approximation of the rectus abdominis [4]. Additional mobilization is achieved by release of the posterior rectus sheath from the rectus muscle. Studies with reliable and lengthy follow-up show 
high reherniation rates up to $37.7 \%$ after large ventral hernia repair after CST without mesh [5] While retrospective studies demonstrate a lower reherniation risk when mesh reinforcement is used, there are no studies comparing reherniation risk head to head with or without use of mesh [6, 7]. In the literature, risk factor analysis for postoperative complication and reherniation has focused almost exclusively on patient history and co-morbidity (e.g., obesity, smoking, previous reherniation). While surgeons heavily rely on physical exam and CT scans, CT scan measurements have not been extensively studied to predict adverse outcomes $[8,9]$.

Computed tomography (CT) scans are more and more routinely performed during patient workup for adequate preoperative planning of large ventral hernias. While the scans can be used to determine hernia characteristics, more abdominal characteristics can be measured using a dedicated workstation, including the visceral and subcutaneous fat components of the abdomen. It is known that for predicting complications in colorectal surgery, visceral fat volume is more accurate in predicting the risk to the development of an incisional hernia than BMI [10]. Since BMI does not accurately reflect visceral fat mass, it is likely that these results can be translated to other forms of surgery including complex ventral hernia repair.

The aim of the current study is to explore the use of CT scan-derived body morphometrics [visceral fat volume (VFV), subcutaneous fat volume (SFV), total fat volume (TFV) and loss of domain (LOD)] to predict reherniation and SSI in patients undergoing complex ventral hernia repair using the CST. This may allow for a more reliable quantitative risk analysis leading to better-informed decisions regarding efficacy and safety of surgery and inform patients in a patient-tailored fashion.

\section{Patients and methods}

\section{Study population and surgical procedure}

The study was performed following a retrospective cohort design. Electronic patient records were searched for patients who met the inclusion criteria, after which a chart review (using a pre-defined case report form) was performed. Adult patients (18-75 years of age at time of operation) who underwent complex ventral hernia repair using the CST (with or without the use of mesh reinforcement) between 2000 and 2013 and who had a preoperative CT scan were eligible for inclusion. Patients were excluded if the CT scan was performed earlier than 6 months prior to surgery to minimize the influence of changes in weight, or if the scan did not cover the full abdomen. Patient demographics, operation data, the occurrence of reherniation, SSI within 30 days and follow-up information were extracted (Table 1).
Table 1 Peri-operative patient characteristics

\begin{tabular}{lc}
\hline Peri-operative variables & $n(\%)$ \\
\hline Patients & $65(100)$ \\
Male & $49(75.4)$ \\
BMI $\left(\mathrm{kg} / \mathrm{m}^{2}\right)$, median (range) & $26.3(20-37.2)$ \\
Obesity, BMI $>30$ & $12(18.5)$ \\
Age, median (range) & $62(23-78)$ \\
Mesh reinforcement & $45(69.2)$ \\
Vypro mesh & $22(33.1)$ \\
Proceed mesh & $17(25.4)$ \\
Ultrapro mesh & $4(6.2)$ \\
Prolene mesh & $2(3.1)$ \\
Sepra mesh & $1(1.5)$ \\
Operation duration, median (range) & $206.5(26-420)$ \\
Blood loss, median (range) & $750(150-2000)$ \\
Surgical site infection $>30$ days & $14(21.5)$ \\
Reherniation & $18(27.7)$ \\
Follow-up, median (range) & $14(0-82)$ \\
\hline
\end{tabular}

SSI was defined according to the centers for disease control and prevention (CDC) definitions for surgical site infection.

CST was performed as previously described [5]. Briefly, uni- or bilateral release of the external oblique aponeurosis is performed to achieve medial translation of the rectus complex, with or without mobilization of the posterior rectus sheath for additional medialization. The aim is always to close the posterior fascia and also spare the peri-umbilical perforators if possible. A synthetic mesh was used only if there was no concurrent infection during reconstruction of the abdominal wall. If a mesh was used it was placed with an overlap of $5 \mathrm{~cm}$ on each side of the defect. In some patients with a non-contaminated wound, reconstruction without mesh reinforcement was used because of a concurrent trial that was being performed at the time. This study aimed to compare the results of mesh reinforcement in addition to CST and CST without mesh reinforcement. All procedures were performed exclusively by a group of four experienced abdominal wall surgeons.

\section{Computed tomography measurements}

The CT scans were analyzed by the corresponding author (HW) using a dedicated workstation (Aquarius 3D Workstation, TeraRecon, San Mateo, CA, USA), which was able to separate intra- from extra-abdominal fat. Fat volumes were analyzed using a modified measurement protocol described by Rickles et al. [11]. The VFV, SFV and waist circumference (WC) were measured semi-automatically every $1.2 \mathrm{~cm}$ up to $12 \mathrm{~cm}$ cranially from the most cranial slice in which S1 was still visible. The range of Hounsfield units (HU) to determine adipose tissue was between -150 and 


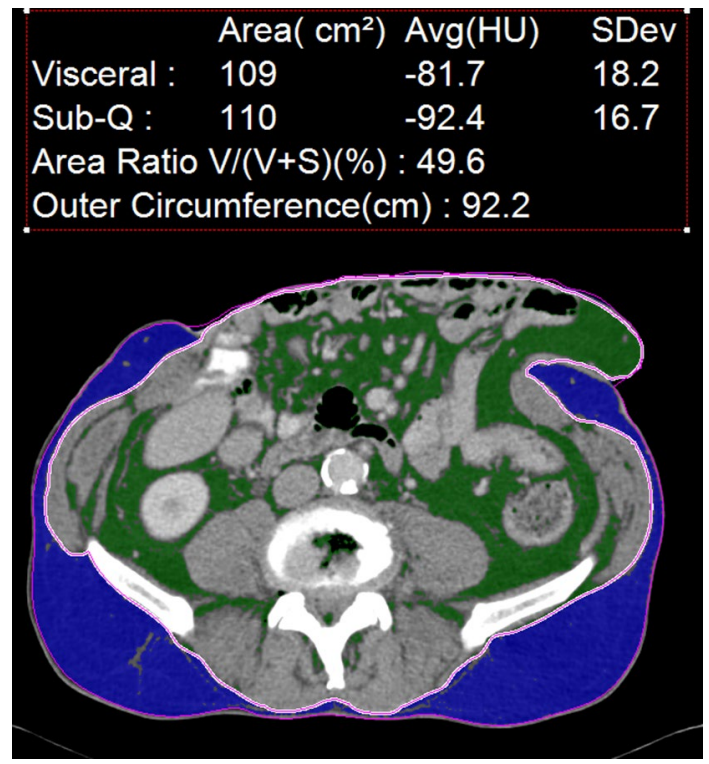

Fig. 1 Quantification of the visceral and subcutaneous fat. The green and blue sections represent the visceral and subcutaneous fat, respectively. (Color figure online)

-50 . Measurements were manually corrected if the intraabdominal area was not correctly demarcated from the extraabdominal area (Fig. 1). All measurements were systematically re-evaluated by HW, NJS and SH and differences were solved by consensus.

Rectus abdominis muscle thickness and width were measured at L2-4 level. Sagittal diameter (SD: the distance from the corpus vertebrae to the internal boundary of the abdominal wall) and WC were measured at the umbilical level (at the intervertebral disc between L3 and L4; Fig. 2). To determine LOD, a 3D reconstruction was made from the abdomen by tracing the abdomen and hernia sac from the pubic bone

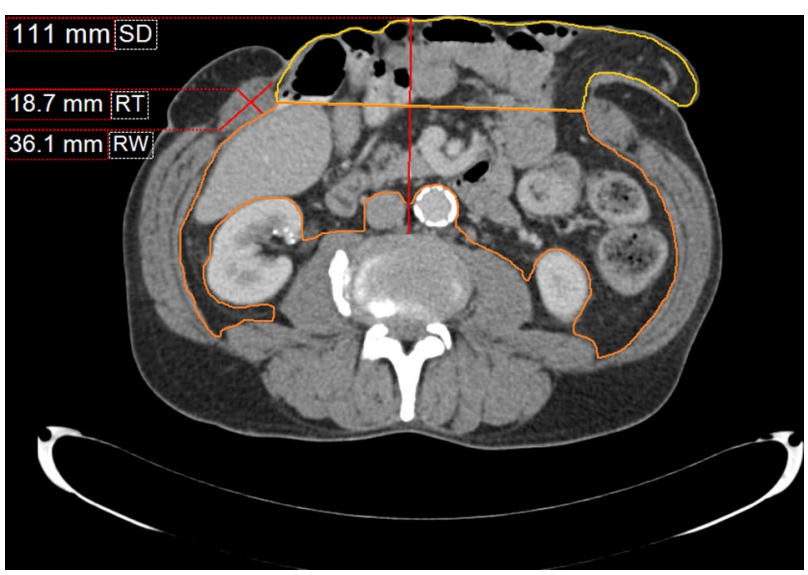

Fig. 2 CT measurements. The orange outline represents the intraabdominal area whereas the yellow outline represents the hernia sac. $\mathrm{SD}, \mathrm{RT}$ and RW can be seen on the left. (Color figure online)

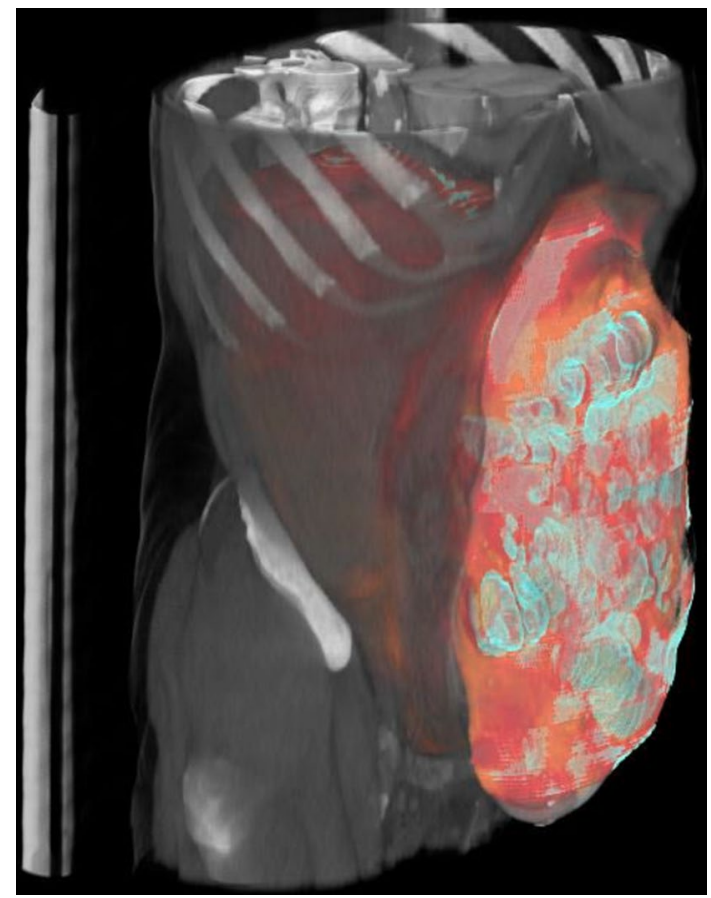

Fig. 3 3D reconstruction of the abdomen. The colored part represents the hernia and the abdomen which were used for calculation of segmentation volumes of the abdomen and hernia sac. (Color figure online)

to the diaphragm using at least 20 manual measurements. The intermediate measurements were calculated by the software and then manually checked for accurate separation of the intra-abdominal and extra-abdominal area. If necessary, manual correction was performed for each slice of the scan. Abdominal volume, hernia sac volume and LOD were calculated based on these measurements (Fig. 3). Measurement results are presented in Table 2.

\section{Statistical analysis}

For all measured variables, a Cox regression analysis was performed with reherniation as outcome and a univariate binary logistic regression was performed with SSI as outcome. Variables were included in multivariate Cox regression with reherniation as outcome and binary logistic regression analysis with SSI as outcome if the $p$ value was $<0.2$ in univariate analysis. To prevent the inclusion of highly correlating variables in multivariate Cox and logistic regression analysis, the Pearson correlation coefficients between variables were determined. Out of CT scan measurements with a Pearson correlation coefficient $>0.7$ the one with the lowest $p$ value was entered in the multivariate analysis [12]. Variables with a $p$ value $<0.05$ were considered significant in multivariate analysis. 
Table 2 Computer tomography measurements

\begin{tabular}{lcr}
\hline Computer tomography measurements & Mean (range) \\
\hline Rectus thickness $(\mathrm{mm})$ & $14.17(7.09-51.90)$ & $54.78(20.20-133.13)$ \\
Rectus width $(\mathrm{mm})$ & $8937(3698-13,983)$ & $658(0-2600)$ \\
Abdominal volume $\left(\mathrm{cm}^{3}\right)$ & $6.82(0.00-29.72)$ & Female \\
Hernia sac volume $\left(\mathrm{cm}^{3}\right)$ & $379.86(87.54-661.18)$ & $226(99.9-419)$ \\
Loss of domain & Male & $143(49.5-310)$ \\
Total fat $\left(\mathrm{cm}^{2}\right)$ & $165(38.2-405)$ & $95.9(81.8-114)$ \\
\hline Subcutaneous fat $\left(\mathrm{cm}^{2}\right)$ & $217(49.4-398)$ & $115(73.8-166)$ \\
Visceral fat $\left(\mathrm{cm}^{2}\right)$ & $102(83.8-120)$ & $138(77.5-200)$ \\
Waist circumference $(\mathrm{cm})$ & & \\
Sagittal distance $(\mathrm{cm})$ & & \\
\hline
\end{tabular}

${ }^{a}$ The distance between the corpus vertebrae and the abdominal wall

Table 3 Missing variable analyses

\begin{tabular}{lc}
\hline Variable & $n(\%)$ \\
\hline BMI & $4(6.2)$ \\
Obese & $4(6.2)$ \\
OK duration & $1(1.5)$ \\
Blood loss & $12(18.5)$ \\
Size defect & $3(4.6)$ \\
Rectus thickness & $4(6.2)$ \\
Rectus with & $6(9.2)$ \\
Hernia volume & $1(1.5)$ \\
Abdominal volume & $2(3.1)$ \\
Loss of domain & $3(4.6)$ \\
Waist circumference & $1(1.5)$ \\
Visceral fat volume & $2(3.1)$ \\
Subcutaneous fat volume & $3(4.6)$ \\
Total fat volume & $2(3.1)$ \\
\hline
\end{tabular}

A scatter plot was created for VFV versus body mass index (BMI) to determine if BMI is a predictor of visceral fat. All analyses were performed with IBM SPSS Version 22 (IBM Corp., Armonk, N.Y.).

\section{Results}

A total of 209 patients were operated for complex ventral hernia using CST between 2000 and 2013. 65 patients had a CT scan performed within 6 months before surgery and both the CT scans and the follow-up were performed at our hospital. Patient demographics and peri-operative variables are described in Tables 1 and 2. Briefly, in our population, reherniation occurred in 18 patients $(27.7 \%)$ and SSI occurred in 14 patients $(21.5 \%)$. The average follow-up (defined as clinical examination in the out-patient clinic) was 14 months (range 0-82). Mesh reinforcement was used in 45 patients $(69.2 \%)$. Mesh was placed in a sublay $(73.1 \%)$, intraperitoneal $(16.2 \%)$, onlay $(7.6 \%)$ or inlay $(1.5 \%)$ position. Missing variables are demonstrated in Table 3.

\section{Reherniation}

The use of a mesh $(p=0.074, \mathrm{OR}=0.42)$, VFV $(p=0.029$, $\mathrm{OR}=1.67)$ and TFV $(p=0.139$, OR $=1.27)$ were predictors for reherniation in univariate Cox regression (Table 4). Mesh reinforcement was a protective predictor of reherniation where an increase of VFV and TFV increased the risk of recurrence. VFV and TFV correlated with $r=0.81$. Since VFV and TFV correlated $>0.7$ and VFV had a lower $p$ value, TFV was omitted from multivariate analysis. Therefore, VFV and the use of a mesh were entered into a multivariate Cox regression analysis with reherniation as outcome.

In the multivariate analysis, only VFV per $1000 \mathrm{~cm}^{3}$ ( $p=0.025, \mathrm{OR}=1.65)$ was a significant predictor for reherniation where an increase in VFV increases the risk of recurrence. The use of mesh reinforcement $(p=0.062$, $\mathrm{OR}=0.15$ ) was not statistically significant as a protective factor in this multivariate model.

\section{Surgical site infection}

Significant predictors of SSI in univariate analysis were female sex $(p=0.119$, OR $=0.19)$, hernia sac volume $(p=0.119, \mathrm{OR}=1.41), \mathrm{LOD}(p=0.128, \mathrm{OR}=1.39) \mathrm{SFV}$ $(p=0.018, \mathrm{OR}=0.31), \mathrm{TFV}(p=0.065, \mathrm{OR}=0.67)$ and number of previous hernia repairs $(p=0.087, \mathrm{OR}=1.58)$. 
Table 4 Univariate risk analysis results

\begin{tabular}{|c|c|c|c|c|c|c|c|c|}
\hline \multirow{2}{*}{\multicolumn{3}{|c|}{ Variable }} & \multicolumn{6}{|c|}{ Univariate OR ( $95 \% \mathrm{CI}), p$ value } \\
\hline & & & \multicolumn{4}{|c|}{ Reherniation } & \multicolumn{2}{|l|}{ Infection } \\
\hline \multicolumn{3}{|l|}{ Sex (female) } & \multicolumn{4}{|c|}{$0.69(0.23-2.10), 0.512$} & \multicolumn{2}{|c|}{$0.19(0.22-1.54), 0.119^{*}$} \\
\hline \multicolumn{3}{|l|}{ BMI } & \multicolumn{4}{|c|}{$1.08(0.94-1.23), 0.240$} & \multicolumn{2}{|c|}{$0.92(0.78-1.09), 0.325$} \\
\hline \multicolumn{3}{|l|}{ Mesh } & \multicolumn{4}{|c|}{0.42 (0.16-1.09), 0.074*,† } & \multicolumn{2}{|c|}{$1.83(0.45-7.46), 0.397$} \\
\hline \multicolumn{3}{|l|}{ SSI } & \multicolumn{4}{|c|}{$0.61(0.18-2.14), 0.443$} & \multicolumn{2}{|l|}{-} \\
\hline \multicolumn{3}{|c|}{ Rectus thickness, per $10 \mathrm{~mm}$} & \multicolumn{4}{|c|}{$3.26(0.42-25.24), 0.258$} & \multicolumn{2}{|c|}{$1.46(0.66-3.20), 0.365$} \\
\hline \multicolumn{3}{|c|}{ Rectus width, per $10 \mathrm{~mm}$} & \multicolumn{4}{|c|}{$1.23(0.75-2.03), 0.419$} & \multicolumn{2}{|c|}{$1.13(0.81-1.58), 0.455$} \\
\hline \multicolumn{3}{|c|}{ Abdominal volume, per $500 \mathrm{~cm}^{3}$} & \multicolumn{4}{|c|}{$1.42(0.82-2.44), 0.213^{*}$} & \multicolumn{2}{|c|}{$0.91(0.71-1.19), 0.755$} \\
\hline \multicolumn{3}{|c|}{ Hernia sac volume, per $500 \mathrm{~cm}^{3}$} & \multicolumn{4}{|c|}{$1.13(0.80-1.59), 0.480$} & \multicolumn{2}{|c|}{$1.41(0.92-2.16), 0.119^{*}$} \\
\hline \multicolumn{3}{|c|}{ Loss of domain, per $5 \%$} & \multicolumn{4}{|c|}{$0.41(0.16-1.09), 0.956$} & \multicolumn{2}{|c|}{$1.39(0.93-1.75), 0.128^{*}$} \\
\hline Waist circumferenc & $\mathrm{cm}$ & & 1.23( & $02)$, & & & $0.96(0.28$ & $.090 *$ \\
\hline Sagittal distance $^{\mathrm{a}}, \mathrm{p}$ & & & 1.08 & 27), & & & $1.05(0.86$ & .603 \\
\hline Visceral fat, per 10 & & & 1.67( & 64), & & & $0.72(0.41-$ & .246 \\
\hline Subcutaneous fat, $\mathrm{p}$ & $\mathrm{cm}^{3}$ & & 1.29( & $09)$, & & & $0.31(0.12$ & $.018^{*, \dagger}$ \\
\hline Total fat, per 1000 & & & 1.27( & 74), & & & $0.67(0.44$ & $.065 *$ \\
\hline Defect size, per $\mathrm{cm}$ & & & 0.99( & $00)$, & & & $1.00(0.99$ & 0.910 \\
\hline Number of previou & & & 1.03 & 42), & & & $1.58(0.94$ & $.087 *$ \\
\hline & $B$ & S.E. & Wald & $d f$ & Sig. & $\operatorname{Exp}(B)$ & $95 \% \mathrm{CI}$ & (B) \\
\hline & & & & & & & Lower & Upper \\
\hline Step $1^{\mathrm{b}}$ & & & & & & & & \\
\hline Sex & -1.983 & 1.571 & 1.592 & 1 & 0.207 & 0.138 & 0.006 & 2.994 \\
\hline v_br_per_500 & 0.187 & 0.763 & 0.060 & 1 & 0.806 & 1.206 & 0.270 & 5.382 \\
\hline lod_per_5 & 0.521 & 0.676 & .0595 & 1 & 0.440 & 1.684 & 0.448 & 6.331 \\
\hline sv_vol_per_1000 & -1.308 & 0.647 & 4.089 & 1 & 0.043 & 0.270 & 0.076 & 0.961 \\
\hline n_prev_repair & 0.882 & 0.522 & 2.859 & 1 & 0.091 & 0.417 & 0.869 & 6.721 \\
\hline Constant & 1.1527 & 1.584 & 0.929 & 1 & 0.335 & 4603 & & \\
\hline
\end{tabular}

${ }^{\mathrm{a}}$ The distance between the corpus vertebrae and the abdominal wall

${ }^{b}$ Variable(s) entered on step 1: sex, v_br_per_500, lod_per_5, sv_vol_per_1000, n_prev_repair

* Significant during univariate analysis

${ }^{\dagger}$ Significant during multivariate analysis

Hernia sac volume and LOD correlated with $r=0.94$. Therefore, LOD was excluded from multivariate analysis. SFV and TFV correlated with $r=0.75$. Therefore, TFV was excluded from multivariate analysis. In multivariate binary logistic regression analysis, hernia sac volume and SFV proved to be significant predictors $(p=0.020$, $\mathrm{OR}=2.10$ and $p=0.034$, $\mathrm{OR}=0.26$, respectively). Number of previous hernia repairs and sex were not significant $(p=0.089, \mathrm{OR}=2.24$ and $p=0.252, \mathrm{OR}=0.239$ respectively).

\section{Fat volume correlations}

BMI and VFV were only weakly correlated $(r=0.45)$, whereas BMI was more strongly correlated to total fat and subcutaneous fat ( $r=0.73$ and $r=0.75$, respectively).

\section{Discussion}

In this study, we demonstrate that reherniation rate after hernia repair using the CST can be predicted by VFV measured on a pre-operative CT scan. For every $900 \mathrm{~g}$ (1.98 lbs) increase of visceral fat, the risk of reherniation almost doubled. In addition, hernia sac volume and SFV are predictors of SSI ( $\mathrm{OR}=2.05$ and $\mathrm{OR}=0.22$, respectively). Interestingly, in our population, an increase of subcutaneous fat mass decreased the risk to develop SSI. These findings suggest that CT measurements are a valuable tool for preoperative risk assessment in patients undergoing complex ventral hernia repair using the CST.

Currently, BMI is used in clinical practice to predict adverse outcomes after hernia repair. However, in our population VFV was a significant predictor of reherniation whereas BMI was not. This may be explained by the poor 
Fig. 4 Scatterplot of BMI

versus different fat volumes demonstrating correlations of $p=0.73, p=0.44$ and $p=0.75$ for total fat versus BMI, visceral fat volume versus BMI and subcutaneous fat volume versus BMI, respectively
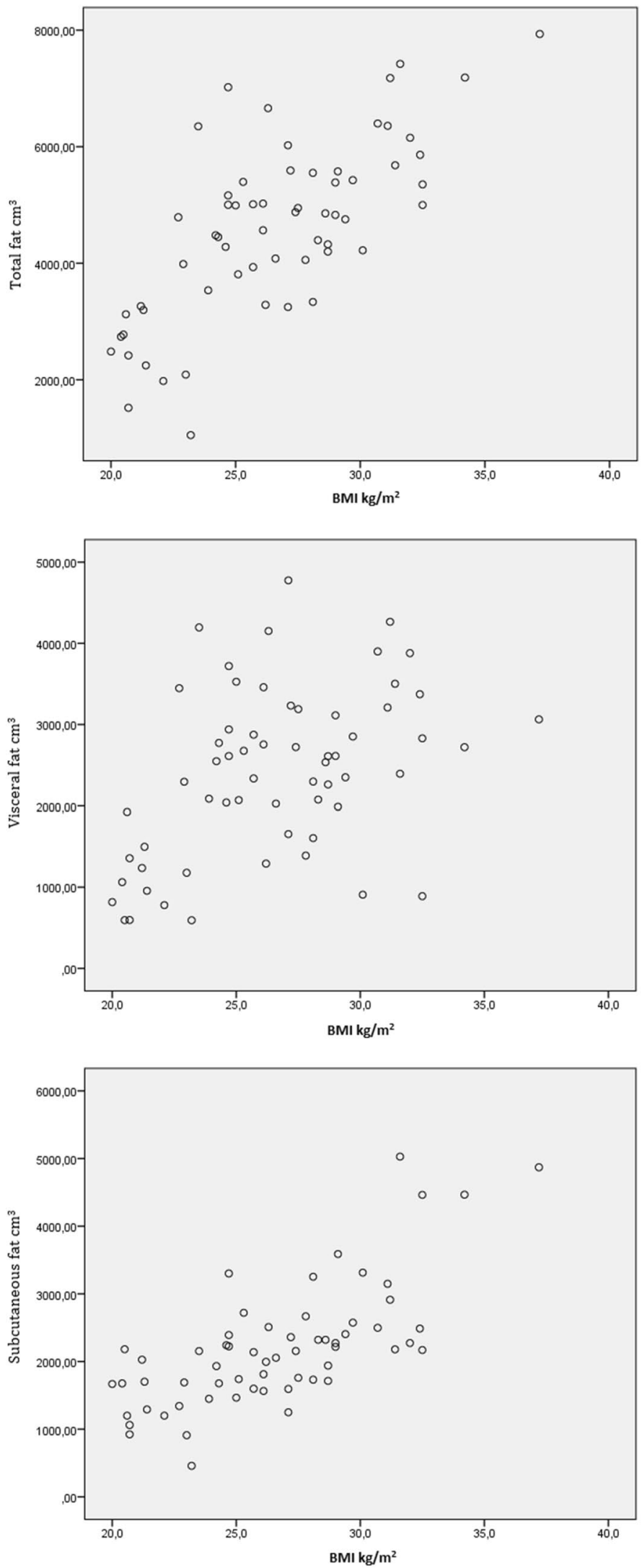
correlation between BMI and visceral fat (Fig. 4). In addition, a recent study found that BMI only increased the risk of reherniation when BMI was over $30 \mathrm{~kg} / \mathrm{m}^{2}$ [13]. Therefore, visceral fat mass may more accurately predict reherniation than BMI.

SFV was significantly associated with a lower incidence of SSI in our study population. Recently, Levi et al. described subcutaneous fat as a risk factor for infection in patients who underwent CST for ventral hernia repair [14]. However, the BMI in our population $\left(26.5 \mathrm{~kg} / \mathrm{m}^{2}\right)$ is substantially lower than in Levi's $\left(33 \mathrm{~kg} / \mathrm{m}^{2}\right)$. Since BMI and SFV seem strongly correlated ( $p=0.75$ in the current study), the possible negative effects of subcutaneous fat on SSI might only become apparent above a certain SFV [15]. Further prospective research could clarify the role of subcutaneous fat for predicting SSI in both obese and non-obese populations.

A major advantage of our study over others is the accuracy in which the fat volumes were determined. Using a volume calculated of multiple CT coupes (in contrast to using a single coupe), and correcting each measurement manually there is a high degree of construct validity [14]. However, this approach was quite time consuming and we are currently working on a proxy that can be measured easily and fast to make it more readily applicable in practice.

A statistical limitation of our study is the relatively small sample size. Our inclusion number is lower than expected, mainly because a CT scan was not available prior to surgery in all patients. Moreover, throughout the years, CT scan protocols had been modified. Therefore, in certain periods, some (most upper and lower) parts of the abdomen were not scanned and these patients had to be excluded from this retrospective analysis.

\section{Conclusion}

Our study indicates that visceral fat volume, subcutaneous fat volume and hernia sac volume derived from CT scan measurements may be used to predict reherniation and SSI in patients undergoing complex ventral hernia repair using CST. With this information, prospective trials may further identify the role of these CT scan-derived body morphometrics for patient-tailored risk assessment. Our findings need confirmation in future prospective studies, preferably multi-centered to allow for greater study sizes and analysis of more homogenous groups.

Author contributions HW, LK and NJS contributed to research design, data extraction and analysis and paper drafting. ORB and EG participated in the research design and paper drafting. SH participated in research design and the setup of measurement protocols. HG and DJOU were part of research design and paper drafting.

\section{Compliance with ethical standards}

Conflict of interest HW declares no conflict of interest. LK declares no conflict of interest. ORB declares no conflict of interest. SH declares no conflict of interest. DJOU declares no conflict of interest. HVG declares no conflict of interest. EVG declares no conflict of interest. NJS declares no conflict of interest.

Ethical approval This study was approved by the institutional review board of the Radboudumc.

Human and animal rights All procedures performed in studies involving human participants were in accordance with the ethical standards of the institutional and/or national research committee and with the 1964 Helsinki declaration andits later amendments or comparable ethical standards.

Informed consent For this type of study, formal consent was not necessary.

Open Access This article is distributed under the terms of the Creative Commons Attribution 4.0 International License (http://creativeco mmons.org/licenses/by/4.0/), which permits unrestricted use, distribution, and reproduction in any medium, provided you give appropriate credit to the original author(s) and the source, provide a link to the Creative Commons license, and indicate if changes were made.

\section{References}

1. Richmond B, Ubert A, Judhan R, King J, Harrah T, Dyer B et al (2014) Component separation with porcine acellular dermal reinforcement is superior to traditional bridged mesh repairs in the open repair of significant midline ventral hernia defects. Am Surg 80(8):725-731

2. de Vries Reilingh TS, van Goor H, Charbon JA, Rosman C, Hesselink EJ, van der Wilt GJ et al (2007) Repair of giant midline abdominal wall hernias: "components separation technique" versus prosthetic repair: interim analysis of a randomized controlled trial. World J Surg 31(4):756-763

3. de Vries Reilingh TS, van Goor H, Rosman C, Bemelmans MH, de Jong D, van Nieuwenhoven EJ et al (2003) "Components separation technique" for the repair of large abdominal wall hernias. J Am Coll Surg 196(1):32-37

4. Ramirez OM, Ruas E, Dellon AL (1990) "Components separation" method for closure of abdominal-wall defects: an anatomic and clinical study. Plast Reconstr Surg 86(3):519-526

5. Slater NJ, van Goor H, Bleichrodt RP (2015) Large and complex ventral hernia repair using "components separation technique" without mesh results in a high recurrence rate. Am J Surg 209(1):170-179

6. Slater NJ, Knaapen L, Bokkerink WJ, Biemans MJ, Buyne OR, Ulrich DJ et al (2015) Large contaminated ventral hernia repair using component separation technique with synthetic mesh. Plast Reconstr Surg 136(6):796e-805e

7. Slater NJ, van der Kolk M, Hendriks T, van Goor H, Bleichrodt RP (2013) Biologic grafts for ventral hernia repair: a systematic review. Am J Surg 205(2):220-230

8. DiCocco JM, Magnotti LJ, Emmett KP, Zarzaur BL, Croce MA, Sharpe JP et al (2010) Long-term follow-up of abdominal wall 
reconstruction after planned ventral hernia: a 15-year experience. J Am Coll Surg 210(5):686-695 (95-8)

9. Ko JH, Wang EC, Salvay DM, Paul BC, Dumanian GA (2009) Abdominal wall reconstruction: lessons learned from 200 "components separation" procedures. Arch Surg (Chicago, Ill: 1960) 144(11):1047-1055

10. Aquina CT, Rickles AS, Probst CP, Kelly KN, Deeb AP, Monson JR et al (2015) Visceral obesity, not elevated BMI, is strongly associated with incisional hernia after colorectal surgery. Dis Colon Rectum 58(2):220-227

11. Rickles AS, Iannuzzi JC, Mironov O, Deeb AP, Sharma A, Fleming FJ et al (2013) Visceral obesity and colorectal cancer: are we missing the boat with BMI? J Gastrointest Surg 17(1):133-143 (discussion $\mathrm{p} 43$ )

12. Tabachnick BG, Fidell LS (1996) Using multivariate statistics, 3rd edn. HarperCollins College Publishers, New York

13. Desai KA, Razavi SA, Hart AM, Thompson PW, Losken A (2016) The effect of BMI on outcomes following complex abdominal wall reconstructions. Ann Plast Surg 76:S295-S297. https://doi. org/10.1097/SAP.0000000000000673

14. Levi B, Zhang P, Lisiecki J, Terjimanian MN, Rinkinen J, Agarwal $S$ et al (2014) Use of morphometric assessment of body composition to quantify risk of surgical-site infection in patients undergoing component separation ventral hernia repair. Plast Reconstr Surg 133(4):559e-566e

15. Kim JH, Doo SW, Cho KS, Yang WJ, Song YS, Hwang J et al (2015) Which anthropometric measurements including visceral fat, subcutaneous fat, body mass index, and waist circumference could predict the urinary stone composition most? BMC Urol 15:17

Publisher's Note Springer Nature remains neutral with regard to jurisdictional claims in published maps and institutional affiliations. 